\title{
Oxidative properties of Moringa oleifera kernel oil from different extraction methods during storage
}

\begin{abstract}
Background: Moringa oleifera (MO) kernel oil is categorized as a high-oleic oil that resembles olive oil. However, unlike olive trees, MO trees are largely present in most subtropical and tropical countries. In these countries, therefore, the benefits of oleic acid can be obtained at a cheaper price through the consumption of MO kernel oil. This study reports on the effect of different extraction methods on oxidative properties of MO kernel oil during storage for 140 days at 13,25 , and $37^{\circ} \mathrm{C}$. Results: All aqueous enzymatic extraction (AEE)-based methods generally resulted in oil with better oxidative properties and higher tocopherol retention than the use of solvent. Prior to AEE, boiling pre-treatment deactivated the hydrolytic enzymes and preserved the oil's quality. In contrast, high-pressure processing (HPP) pre-treatment accelerated hydrolytic reaction and resulted in an increase in free fatty acids after 140 days at all temperatures. No significant changes were detected in the oils' iodine values and fatty acid composition. The tocopherol content decreased significantly at both 13 and $25^{\circ} \mathrm{C}$ after 60 days in the oil from SE method, and after 120 days in oils from AEE-based methods. Conclusion: These findings are significant in highlighting the extraction methods resulting in crude MO kernel oil with greatest oxidative stability in the storage conditions tested. Subsequently, the suitable storage condition of the oil prior to refining can be determined. Further studies are recommended in determining the suitable refining processes and parameters for the MO kernel oil prior to application in variety food products. (C) 2019 Society of Chemical Industry.
\end{abstract}

Keyword: Drumstick; Fat; Horseradish; Lipid; Murunggai; Seed 